\title{
Desarrollo de la conciliación a partir de la Constitución de 1991
}

\author{
PABLO TOMÁS SILVA MARIÑO \\ Fecha de recepción: 30-06-2009 - Aprobación: 11-08-2009
}

\section{Resumen}

En este artículo se busca dar una mirada a los métodos alternativos de solución de conflictos (MASC), entre los cuales se destaca la conciliación, que por su utilidad, historia legislativa y eficacia en el esquema de sociedad y economía del Estado colombiano constituye una materia interesante y amena de estudiar.

La evolución e historia legislativa de figuras como la conciliación son tan antiguas como nuestra historia de nación independiente, si bien los MASC solo han logrado obtener su reconocimiento y espacio a partir de la expedición de la Constitución Política de 1991, con su polémico artículo 116 que permite a los particulares, de manera transitoria, administrar justicia, y sobre el cual se ha presentado todo un desarrollo legislativo en el que se destacan las leyes 446 de 1998, 640 de 2001 y la reciente 1285 de 2009.

Por su parte, el Ministerio del Interior y de Justicia es el vigilante y supervisor de las distintas figuras que componen los MASc, además de articular todo el engranaje que implica la figura de la conciliación, tal y como fue concebida por las normas antes señaladas. Asimismo, esa cartera se ha encargado de recolectar y tabular todas las cifras arrojadas por la aplicación de la conciliación, las cuales sin duda alguna permiten la evaluación de la efectividad y utilidad de la misma.

Con base en las cifras estudiadas y puestas en el contexto histórico -y nutridas con la experiencia del autor- se procura llegar a una serie de conclusiones y elaborar algunas recomendaciones con miras a fortalecer y afianzar tanto la conciliación como las otras figuras afines que constituyen los MASC.

\section{Palabras clave}

Métodos alternativos de solución de conflictos, MASc, conciliación, centros, conciliadores, judicantes, estadísticas, solución directa, abogados, cultura, sociedad, materias conciliables, actas, constancias, educación y cifras.

\section{Abstract}

The alternative conflict solution methods (MASc in Spanish) are an interesting and entertaining subject of study. These methods are recognized by their utility, legislative history, and efficiency in the Colombian State, society, and economy.

Figures with a legislative history and evolution as old as our history as an independent nation, and have been recognized only from the Political Constitution of 1991 through its polemic article 116, which allows particulars to temporarily manage justice. According to this, there has been a complete legislative development, highlighting laws 446 of 1998, 640 of 2001, and the most recent, 1285 of 2009.

The Ministry of Law and Justice as the supervisor of all the machinery involved in the reconciliation as it was conceived by the laws previously mentioned. In addition, it has been in charge of gathering and tabulating all the figures resulting from applying the reconciliation. These figures allow making several conclusions with respect to its effectiveness and utility.

Finally, based on the figures studied and putting them in a context -and the author's experience- leads him to conclude and recommend several ideas in order to strengthen and consolidate not only the reconciliation, but also the other figures that are part of the MASC.

\section{Key words}

Alternative conflict solution methods, MASc, reconciliation, centers, judge, statistics, direct solution, lawyers, culture, society, reconcilable goods, minutes, certificates, education, and figures. 


\section{INTRODUCCIÓN}

En 1998, cuando una corriente de abogados y algunos otros profesionales de las ciencias sociales buscaban una línea nueva de especialización, los métodos alternativos de solución de conflictos (MASc) se presentaban como una posibilidad extraña, por no decir exótica, de intensificación del conocimiento y desarrollo de la profesión, situación que tan solo una década después de aquellos días puede resultar inverosímil, ya que puede afirmarse que hoy no existe conflicto llamado a resolverse por un proceso judicial, que no intente, o por lo menos no contemple, la posibilidad de un arreglo a partir de un método alternativo de solución de conflictos, en especial por medio de la conciliación.

Pues bien, aquel grupo que tomó la alternativa de intensificar y desarrollar su profesión en los MASC, rápidamente se dio cuenta de que había abordado el vehículo correcto, en el momento indicado, y que, si bien el destino del mismo no era conocido, había una gran posibilidad de llegar a un puerto que les permitiese lograr sus metas profesionales, intelectuales y de servicio a la comunidad.

La anterior sensación, se materializó con la promulgación, el 7 de julio de 1998, de la Ley 446, norma que sin lugar a dudas se constituyó en la cosecha de la idea que tímidamente sembró el constituyente cuando estableció en el párrafo $4 .^{\circ}$ del artículo 116 de la Constitución Política de Colombia (сP) de 1991, con relación a la administración de justicia, lo siguiente:

Los particulares pueden ser investidos transitoriamente de la función de administrar justicia en la condición de jurados en las causas criminales, conciliadores o en la de árbitros habilitados por las partes para proferir fallos en derecho o en equidad, en los términos que determine la ley.

Y si bien calificamos de tímido el contenido transcrito en el párrafo anterior de la carta mayor, por la ubicación y la forma como se asentó en el texto de Constitución, no por ello es menos importante, coyuntural y progresista, al modificar el paradigma del monopolio en la administración de justicia en cabeza única y exclusivamente del poder público del Estado, en especial la rama judicial, por la política estatal que adiciona y permite la posibilidad de que los particulares, de manera transitoria, administren justicia a sus pares, en lo que constituye un claro desarrollo del concepto del Estado social de derecho (artículo $1 .^{\circ}$ de la cP) y de los principios de igualdad de las personas (artículo 13 de la сP) y autonomía de la voluntad de los particulares (artículo 6. ${ }^{\circ}$ de la $\mathrm{CP}$ ).

En este orden de ideas, el presente escrito aspira a comentar a los estudiantes, profesores y comunidad académica en general el desarrollo de los MASC en Colombia a partir de la Constitución Política de 1991, habida consideración de la evolución normativa, el impacto en la sociedad y la prospectiva de los mismos, utilizando como eje principal la figura de la conciliación.

\section{Antecedentes en Colombia}

Contraria a la creencia normal, la conciliación no es una figura moderna en el Derecho colombiano, si bien se insiste en el hecho de que solo a partir del artículo 116 de la Constitución Política de Colombia de 1991 renace la conciliación, cuando el constituyente crea un camino que le permite viabilidad jurídica y aceptación social; lo cierto es que la misma existe por lo menos desde la Ley 13 de 1825, promulgada por Francisco de Paula Santander. Esta norma, a lo largo de sus catorce artículos, desarrolló la figura y, desde esa época, hace casi doscientos años, estableció la conciliación como requisito de procedibilidad para acudir a la mayoría de los tribunales judiciales.

Con posterioridad, los laboralistas innovadores, en busca de nuevas opciones, retrotrajeron la ya olvidada conciliación, en la Ley 120 de 1921, por medio de la cual se aplicó a los conflictos colectivos, de manera meramente voluntaria; norma a la cual siguieron los decretos 2158 de 1948, 2663 y 3743 de 1950, que buscaban establecer la conciliación en distintas instancias procesales. A estos avances los sucedieron los decretos 2279 de 1989, cuyos artículos 49 y 50 establecieron la posibilidad de conciliación extrajudicial en las controversias susceptibles de transacción, y el 2282 del mismo año, que instauró la conocida conciliación del artículo 101 del Código de Procedimiento Civil, con algún tinte de obligatoriedad, aunque en la práctica casi siempre operó como mero trámite.

Luego, la Ley 23 de 1991, con un estudio e implementación más juiciosos de la figura y con el propósito de descongestionar los despachos judiciales, reglamentó los centros de conciliación extrajudicial y legalizó los trámites conciliatorios que aisladamente realizaban las 
cámaras de comercio de todo el país. En el mismo sentido, creó la conciliación en equidad, con normas que aún hoy tienen aplicación y cuyo debate y promulgación se hicieron ad portas de los de la nueva constitución.

El último párrafo del artículo 116 de la Carta de 1991 conforma un piso jurídico y un marco constitucional para los MASC, muy especialmente para la conciliación. En desarrollo de esta posibilidad y alimentada por la necesidad del gobierno de turno de buscar una solución para descongestionar los despachos judiciales, colapsados por procesos sin resolver que generaban una lógica sensación de injusticia en la sociedad, se expidió el Decreto 2651 de 1991, que establecía un desordenado régimen de conciliación, en ejercicio de los poderes otorgados por el artículo $5 .^{\circ}$ transitorio de la nueva Constitución. Este decreto, creado para durar 24 meses, fue prorrogado en varias oportunidades hasta llegar a la expedición de la Ley 446 de 1998.

Con la promulgación de la Ley 446, en 1998, llegamos a lo que se puede denominar el inicio de la época moderna de los métodos alternativos de solución de conflictos en Colombia, y naturalmente de la conciliación, ya que esta norma recogió y le dio carácter de permanencia a las contenidas en el citado Decreto 2651 de 1991 y la Ley 23 de 1991; asimismo, subrogó y reguló nuevos aspectos como la conciliación en materia contencioso administrativa y de familia, los conciliadores, los centros de conciliación y la conciliación en equidad, además de tocar temas sobre el procedimiento arbitral y regular la figura de la amigable composición, en los artículos 130 a 132.

En cumplimiento de lo señalado por el artículo 166 de Ley 446, inmediatamente después se expidió el Decreto 1818 de 1998 , el cual intentó compilar en un solo ordenamiento todas las normas referentes a los MASC y cuyo nombre fue (o es, ya que la doctrina no se ha podido poner de acuerdo en su vigencia) Estatuto de los mecanismos alternativos de solución de conflictos, que, una vez revisado por el Consejo de Estado, fue declarada su improcedencia por encontrar que dicho decreto había compilado normas derogadas o subrogadas, así como había dejado por fuera normas vigentes ${ }^{1}$.

Comprobadas las bondades de los MASc, entre los cuales se destacaba la conciliación, con la cual se habían resuelto miles de conflictos en todas las materias, y en consideración a que ello redundó de inmediato en las cifras de descongestión de los juzgados y en los niveles de justicia y satisfacción de la sociedad -que había encontrado en la conciliación una alternativa para solucionar sus conflictos y no de otra manera que hubiese requerido altas inversiones en tiempo y dinero o lo que es peor mediante el empleo de vías de hecho, es decir del empleo de la violencia-, ya en el nuevo siglo se expidió la Ley 640 de 2001, norma que organizó de manera sistemática todos los aspectos de la conciliación, tales como los conciliadores, los centros de conciliación, la clasificación de las conciliaciones, las materias conciliables y, lo más relevante, estableció la conciliación como requisito de procedibilidad para acudir ante la jurisdicción ordinaria.

Con posterioridad a la Ley 640 todos los ordenamientos y normas mayores del país que han regulado situaciones específicas del Derecho o la sociedad, han incorporado un capítulo dedicado a la solución de conflictos de forma directa mediante la utilización de los MAsc, en especial la conciliación, para lo cual basta citar la nueva Ley 1285 de 2009 y su decreto reglamentario 1716 de 2009, por medio de los cuales se regula la conciliación en materia de lo contencioso administrativo y se establece como requisito de procedibilidad para acudir ante la jurisdicción contencioso administrativa el de haber agotado la conciliación previa, salvo excepción expresa de ley.

\section{Cifras y estadísticas de la figura de la conciliación}

Si bien consideramos que hoy son palpables en la sociedad los beneficios de la conciliación y los demás métodos alternativos de solución de conflictos, y deben

\section{PABLO TOMÁS SILVA MARIÑO}

Abogado egresado de la Universidad Externado de Colombia; especialista en Métodos Alternativos de Solución de Conflictos y Negociación de la Universidad del Rosario y diplomado en bases empresariales de la Universidad de la Sabana. Tiene formación para conciliador ante el Ministerio de Justicia y del Derecho de la Cámara de Comercio de Bogotá. Estudió idioma inglés en Stanton School of English, en Londres. Es director de la División Códigos de la Editorial Temis; profesor del módulo marco regulatorio del mercado público de valores, de la cátedra de métodos alternativos de solución de conflictos y negociación de la Universidad Politécnico Grancolombiano y miembro de la lista de conciliadores de la Cámara de Comercio de Bogotá.

ptsilvam@poligran.edu.co

1 Juan Alberto Polo Figueroa fue el magistrado ponente del Consejo de Estado, Sala de lo Contencioso Administrativo, sección primera, radicado 5191 de 1999. 
ser muy pocos los escépticos que aún defiendan la solución de controversias exclusivamente ante los despachos judiciales, consideramos necesario analizar las cifras que al respecto tiene el Ministerio de Justicia y del Derecho, en aras de verificar si este ambiente favorable hacia los MASc tiene un respaldo cuantitativo en la realidad.

Se debe indicar, inicialmente, que en 1998 el número de trámites anuales de conciliación en Colombia superaba con dificultad los mil, cifra que a partir de ese año y hasta 2003, inclusive, creció a un promedio anual del $100 \%$, hasta llegar a la importante cifra de más de 70.000 trámites.

Pues bien, de acuerdo con los datos de Ministerio del Interior y de Justicia, organismo encargado de vigilar, controlar y llevar las estadísticas referentes a la conciliación, se puede establecer que en el año 2002 se realizaron en los diferentes centros de conciliación en Colombia al menos 29.483 trámites, en materias comercial, civil y de familia, de los cuales se conciliaron 11.784 casos; es decir, que aproximadamente se resolvió el 40\% de los casos tramitados.

Este resultado es relevante, aunque solamente representa una tercera parte de los asuntos que en ese mismo año se sometieron ante los tribunales judiciales en el país, pues para esa época, es decir tan solo diez años después de haber aparecido el artículo 116 en la nueva Constitución y con un año de vigencia parcial de la Ley 640 de 2001, ya se solucionaban en Colombia mediante conciliación más de 10.000 conflictos al año, aparte de un número igual de casos que tuvieron una oportunidad previa de solución, antes de llegar ante los estrados judiciales.

El número de trámites o solicitudes de conciliación siguió su crecimiento hasta el año 2004, cuando llegó a 76.060 trámites, con 28.099 acuerdos de conciliación registrados, equivalentes al 36\% de asuntos resueltos (Ministerio del Interior y de Justicia), cifra de "efectividad" que, si bien es más baja que la de 2002 (40\%), tiene su explicación en el hecho de que un gran porcentaje de los trámites efectuados en 2004 se hicieron en cumplimiento de la obligación de agotar la conciliación como requisito de procedibilidad para acudir ante la jurisdicción, pero que en todo caso excede ampliamente el número de casos conciliados en 2002; es decir, resueltos para las partes y la sociedad en general.
Al parecer, de acuerdo con el esquema actual, la conciliación como mecanismo alternativo de solución de conflictos ha alcanzado su máximo nivel, si se tienen en cuenta las cifras de los últimos cuatro años:

- Año 2005, 65.386 trámites iniciados y 23.316 acuerdos de conciliación

- Año 2006, 66.765 trámites iniciados y 24.744 acuerdos de conciliación

- Año 2007, 53.830 trámites iniciados y 20.352 acuerdos de conciliación

- Año 2008, 57.418 trámites iniciados y 23.410 acuerdos de conciliación.

Con base en estos guarismos se concluye que para la última época hay un promedio aproximado de 60.000 trámites iniciados y 23.000 acuerdos, equivalentes al $38 \%$ de conflictos resueltos.

Con respecto a las anteriores cifras es necesario aclarar que el número o porcentaje de acuerdos de conciliación que se ha alcanzado no es un indicador de la efectividad de la figura, ya que quienes tenemos la conciliación como oficio sabemos que existen algunos conflictos no susceptibles de solucionarse en el escenario de la conciliación, pero, por otra parte, aunque no se llegue a una posibilidad de acuerdo, en el 25\% de los casos, en promedio, se ha ganado claridad para las partes en cuanto a su situación jurídica y la posibilidad de éxito de sus pretensiones, que las llevan a tomar una decisión correcta con referencia a las acciones o procesos a iniciar ante la rama judicial.

En sentido contrario, aún existe cerca del 25\% de los trámites que se resuelve por la no asistencia de las partes, con constancia de inasistencia, situación que es un constante dolor de cabeza para los centros de conciliación y los conciliadores, que no logran notificar de la citación para la audiencia de conciliación a las partes, o las partes simplemente desatienden el llamado del conciliador, hecho por el cual el legislador debe tomar medidas como la de endurecer las sanciones por inasistencia, reglamentar los criterios que permiten justificar la inasistencia y, por parte del Estado en general, mejorar sus sistemas de nomenclatura de los predios y ubicación de los ciudadanos.

El 10\% de los trámites de conciliación iniciados, que falta por comentar, hace referencia a los llamados "otros resultados", según las estadísticas del Ministerio del 
Interior y de Justicia, que no son más que los trámites que no son competencia de un determinado centro de conciliación o cuando se trata de aquellos asuntos que por excepción no son materia de conciliación, casos en los cuales se expide la correspondiente constancia, en los términos del artículo 2. ${ }^{\circ}$ de la Ley 640 de 2001. También se registran en esta categoría los asuntos en los que las partes llegan a un arreglo directo por fuera del escenario de la conciliación o no desean seguir adelante con ella, evento en el cual se cancela el trámite.

Con referencia al número de centros de conciliación, se debe recordar que fueron las cámaras de comercio de las principales ciudades del país, en cabeza de la Cámara de Comercio de Bogotá, las que iniciaron la implementación y desarrollo de la conciliación y el arbitraje, principalmente, como métodos alternativos de solucionar conflictos, a finales de la década de los ochenta del siglo pasado, y cómo ellas mantuvieron esta lucha y recomendaron a los legisladores su legalización. Por ende, son en gran parte las precursoras de que hoy existan en el país más de 300 centros de conciliación de todas las categorías, dedicados a las más diversas materias y asuntos.

En efecto, de acuerdo con las cifras oficiales de la entidad de control, se tiene que para el año 2001 ya había en Colombia 111 centros de conciliación registrados, cifra apreciable si se tiene en cuenta que para la expedición de la Ley 446 de 1998 no pasaba de la docena el número de centros de conciliación que operaban en el país; en el año 2002 se incrementó en $35 \%$ el número de centros, hasta llegar a 141 centros registrados y aprobados, crecimiento que se mantuvo constante hasta contar en 2008 con 310 centros de conciliación registrados y aprobados por la cartera de justicia y el Derecho.

La datos antes mencionados permiten concluir, inicialmente, que las organizaciones con y sin ánimo de lucro han escuchado las políticas de Estado por las cuales se hizo el llamado a implementar y ampliar la figura de la conciliación; en segunda instancia, se tiene que reconocer que en la conciliación se ha generado un nicho económico interesante en el que intervienen los centros de conciliación y los conciliadores, y que en muchas ocasiones resulta benéfico para los usuarios; es decir, las personas en conflicto. Por último, es claro que la sociedad ha encontrado en los centros de conciliación un escenario amistoso y agradable para solucionar sus conflictos, diferente del habitual ambiente acartonado $\mathrm{y}$ formalista de los juzgados y tribunales nacionales.
En lo referente a las materias objeto de conciliación se establece que el mayor porcentaje, $70 \%$, corresponde a asuntos en materias civiles y comerciales, en los cuales los conflictos originados en accidentes de tránsito son los más comunes; después se encuentran los asuntos en materia de familia, con el 20\%; los asuntos en materias especiales, como asuntos laborales, administrativos y de patentes, entre otras, corresponden al $8 \%$ y, finalmente, los asuntos en materia penal al $2 \%$.

Sobre los conciliadores es necesario precisar que se clasifican en conciliadores en Derecho y en equidad. Unos de otros se distinguen en que los primeros deben ser necesariamente abogados titulados, mientras que los segundos responden más a características especiales de orden social o cultural, pero las dos clases de conciliadores deben haber aprobado los respectivos cursos de capacitación, estar inscritos en los centros de conciliación y haber obtenido el código del Ministerio del Interior para actuar como tales. El simple hecho de haber cursado y aprobado el curso no convierte a la persona en conciliadora, sino que requiere la inscripción en el centro de conciliación y la autorización del ministerio, salvo la excepción hecha a los estudiantes de los consultorios jurídicos de las universidades y los aspirantes al título de abogado que realizan la judicatura como conciliadores.

Efectuada la anterior precisión, se señala que el Ministerio del Interior reporta 6.225 conciliadores inscritos en el año 2008, 8.350 estudiantes inscritos en los consultorios jurídicos de las distintas universidades del país y 235 judicantes, para un total aproximado al año de 14.800 conciliadores, distribuidos por toda la geografía de Colombia, aunque más de la mitad está ubicada en Bogotá, Medellín y Cali, lo cual tiene su explicación principal en que el mayor número de trámites corresponde a asuntos comerciales, pero con una concentración negativa, pues tan solo 82 municipios, de los más del 1.100 del país, cuentan con un centro de conciliación (El Tiempo, 2008).

En este análisis estadístico, finalmente, es procedente señalar que el Ministerio reporta más de 11.000 capacitados en conciliación por parte de las 89 entidades avaladas para brindar capacitación en conciliación en Derecho y en equidad, cifra que corresponde al $5 \%$, aproximadamente, de los abogados inscritos ante el Consejo Superior de la Judicatura. En otras palabras, se puede interpretar que de cada cien abogados, tan solo cinco tienen una formación como conciliadores, situación que requiere de un análisis profundo para conocer la 
repercusión que esto pueda tener en la administración de justicia y el desarrollo del país en general.

\section{Conclusiones y recomendaciones}

Las cifras expuestas, analizadas a partir de la existencia operativa de la conciliación, relativamente reciente en la línea histórica del Derecho colombiano, permiten afirmar que la implantación de la conciliación como método alternativo de solución de conflictos hecha por el constituyente en la Constitución de 1991 ha sido un éxito. Por una parte, ha logrado modificar la forma como los profesionales del Derecho preparan y afrontan la solución de las controversias, y, por otra, ha logrado en alguna medida descongestionar los despachos judiciales, economizar tiempo y dinero en la solución de las controversias a los ciudadanos, y, como consecuencia de una y otra situación, ha generado una mejoría en la sensación de justicia que tienen los asociados.

Desde el desarrollo científico de los métodos alternativos de solución de conflictos, en especial la conciliación, se puede afirmar que estos han logrado su propia autonomía y lugar en la legislación, la jurisprudencia y la doctrina, con espacios específicos en la academia y en los currículos de las diferentes facultades de ciencias sociales, muy especialmente en las facultades de Derecho, además de dictarse como una asignatura obligatoria en los colegios, sin perjuicio de las afirmaciones románticas de los tratadistas en el tema que afirman que la "conciliación no es una ciencia sino un arte" y que, por ende, su enseñanza corresponde más al área artística que a la ciencia.

Pero su influencia se refleja también en la economía. Los MASC se han introducido en el ejercicio de la profesión de los abogados de manera determinante, tanto por la necesidad de agotar el requisito de procedibilidad para acudir ante los tribunales o juzgados del país, como por la posibilidad que ofrecen de poder solucionar conflictos de forma rápida, certera y económica o de poder esclarecer aquellos que difícilmente pueden atender con prontitud los atareados funcionarios de la administración de justicia. En ese sentido, la conciliación se ha convertido en una práctica constante de importantes sectores económicos como el de la construcción, los servicios públicos domiciliarios, el financiero, las aseguradoras y la finca raíz, entre otros.

De otra parte, la sociedad, sin duda alguna, entendió la profundidad del mandato constitucional otorgado en el artículo 116 y cómo, a partir del ejercicio de la autonomía de la voluntad de cada individuo, es posible solucionar sus conflictos de forma directa, rápida y económica, sin necesidad de acudir a la mano de abogados y con la posibilidad de solucionar las diferencias sin romper las relaciones familiares, sociales y comerciales, que se verían despedazadas si se diera inicio a la acción por la vía judicial.

En el mismo sentido, ha sido acogida claramente la figura de la conciliación como solución a las necesidades de la sociedad colombiana y su aprobación a la forma en que el Estado social de derecho le otorga al individuo un mayor rango en el ejercicio de la autonomía de la voluntad, tal y como se puede verificar en las cifras analizadas.

Sin embargo, no es tiempo de cantar victoria por el crecimiento exponencial de la conciliación. Este no puede ser excusa para que el gobierno, por intermedio del Ministerio del Interior y de Justicia, baje la guardia, ya que las mismas cifras muestran un estancamiento en los últimos años, que si bien puede responder al total cubrimiento de los sectores comunes de conflicto de la sociedad colombiana, como son las materias civiles, comerciales y de familia, queda pendiente la profundización en sectores específicos como los de minería, agricultura y ganadería, curiosamente siempre descuidados, pese a las características socioeconómicas de Colombia.

En materia de educación es necesario insistir en los colegios en la cátedra de la solución directa y amigable de los conflictos, con miras a generar su necesidad y toma de conciencia en las nuevas generaciones de la sociedad, y, de forma más especializada, en las universidades, con importantes esfuerzos, como el del Politécnico Grancolombiano, que ofrece e intensifica en sus programas académicos las áreas de negociación y solución alternativa de conflictos.

Para terminar, es recomendable, a partir de la experiencia positiva que ha brindado la conciliación, insistir y profundizar en figuras afines como son la amigable composición, la mediación, los jueces de paz y la evaluación neutral de casos, que, junto con el arbitraje, ya más extendido, pueden ofrecer todo un ramillete de posibilidades para resolver los conflictos de forma oportuna, expedita y eficaz, que redunda en beneficios para la sociedad, la economía y el propio aparato judicial. 


\section{BIBLIOGRAFÍA}

El Tiempo. No hay certeza sobre cuántos municipios tiene actualmente Colombia. El Tiempo. Obtenido el 21 de Abril de 2008, de www.eltiempo.com/archivo/documento/ CMS-41112512008.

Fisher, Roger; URY, Willian; Patton, Bruce. (1996). Si... De acuerdo. Grupo Editorial Norma.

López Blanco, Hernán Fabio. (1992). Comentarios al decreto de descongestión judicial. Edición ABC.

Ministerio del Interior y de Justicia. (2009). Evaluando las fuentes electrónicas. Disponible en: www.conciliacion.gov.co/paginas_detalle. (Recuperado: 5 de junio de 2009).

Orozco Villegas, Angélica María. (2002). Conciliación, mecanismo alternativo de solución de conflictos por excelencia. Facultad de Ciencias Políticas, Pontificia Universidad Javeriana.

Vargas, Luis Arturo. (1995). La conciliación: avance o retroceso procesal. Revista Foro Universitario. Universidad de Nariño. 